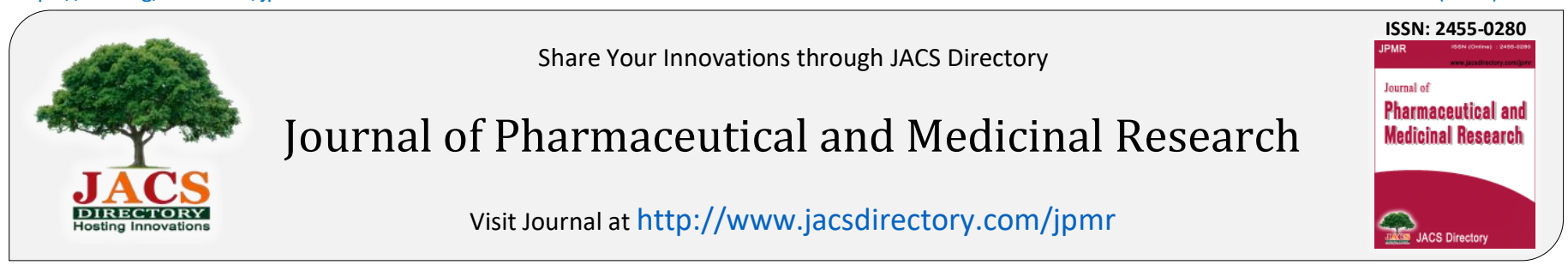

\title{
Assessment of the Likelihood of a Radiation-Induced Abortion in the Unintentional Irradiation of a Live Human Fetus
}

\author{
Eduardo Galiano ${ }^{1, *}$, Marcelo Godin²
}

${ }^{1}$ Dept. of Physics, Laurentian University, Sudbury, ON P3E 2C6, Canada

2Radiation Therapy Service, Hospital Nacional del Cancer "Dr. Manuel Riveros", Asuncion, Paraguay.

\section{ART ICLE D ETA IL S}

\section{Article history:}

Received 13 March 2020

Accepted 17 April 2020

Available online 02 May 2020

\section{Keywords:}

CTDI

Fetal Dose

Radiation-Induced Abortion

Organogenesis

\begin{abstract}
A B S T R A C T
The purpose of this work was to calculate the dose accidentally absorbed by a live fetus during a diagnostic CT procedure on a pregnant patient, and to then assess the likelihood that the premature termination of the pregnancy was radiation-induced. A patient underwent a diagnostic CT procedure as part of her initial clinical workup for a stage II cervical cancer. At the time of imaging - and unbeknownst to the staff - the patient was found to be 12 weeks pregnant. Approximately two weeks later, the fetus became non-viable and was surgically removed. Following established institutional procedures, the case was referred to the physics department for further dosimetric evaluation to determine what role - if any - the fetal dose played in the premature termination of the pregnancy. The fetal dose was determined using Wagner's CTDI Phantom Dose Reference Model method. A slice thickness of $6 \mathrm{~mm}$ and a CTDI $\mathrm{vol}_{\mathrm{vo}}$ of 1.13 mGy were used in our calculations, as suggested in the Abdomen Baby manufacturer's protocol. As suggested by Wagner, KVp, mAs, and slice thickness corrections were applied to the CTDI $\mathrm{vol}_{\text {. }}$. With these parameters, our estimated absorbed dose to the fetus was $19.3 \mathrm{mGy}$. Further, we estimate that the rotation of the fetus through an angle of approximately $90^{\circ}$ along the caudo-cephalic axis during imaging, had no clinically relevant effect on the calculated absorbed dose. The fetal dose was well below the consensus levels for negligible risk (50-150 mGy), and the "actionable" level of $150 \mathrm{mGy}$. At the time of exposure, the fetus was developmentally beyond the critically radiosensitive phase of organogenesis. We conclude that the premature termination of this pregnancy is not likely to be of radiological etiology.
\end{abstract}

\section{Introduction}

In many jurisdictions, existing regulations require that any episode of dose misadministration be properly investigated and documented, in order to address administrative and potential medico-legal issues which may arise. These requirements become paramount in cases involving individuals from the general population who are unintentionally exposed. On a brighter note, these unfortunate episodes present investigators with the possibility of extracting radiobiological data which would be otherwise impossible to obtain due to elemental ethical considerations. In this report, we address the case of a fetus which was accidentally irradiated during a diagnostic CT procedure, and the subsequent clinical evolution of the pregnancy.

The dose to a fetus cannot of course be measured directly, however estimation methods of reasonable accuracy have been developed. Fetal dose estimation methods can be based on physical phantom measurements, or on numerical simulations on virtual phantoms. As examples of the first approach, we cite the works of Felmlee, and Hurwitz, who estimated fetal doses using physical measurements on anthropomorphic phantoms [1, 2]. In terms of numerical approaches, we mention the ImPACT CTDosimetry dose calculator, which is based on Monte Carlo simulations performed by the U.K.'s National Radiological Protection Board with the use of a geometric Medical Internal Radiation Dose (MIRD) phantom model [3-5].

\section{Experimental Methods}

Recently at one of our institutions (H.C., Paraguay), a patient underwent a diagnostic CT procedure. The procedure was performed on a Siemens Somatom Spirit CT unit, as part of the initial clinical workup for a FIGO stage IIB cervical cancer. Upon imaging - and unbeknownst to the staff -

the 34-year-old patient was found to be approximately 12 weeks pregnant, as seen in Figs. 1 and 2.

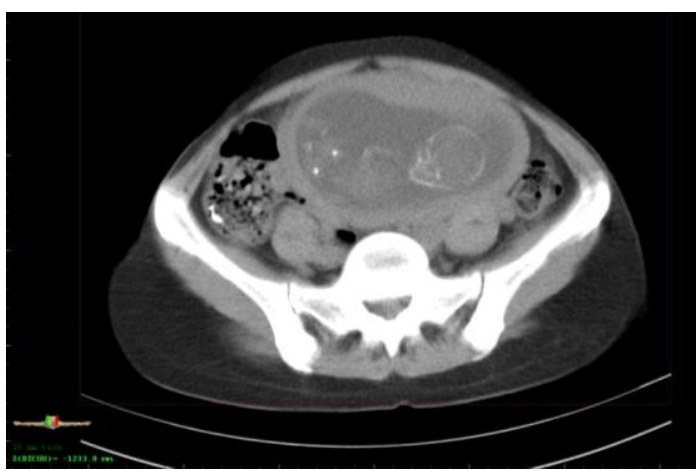

Fig. 1 CT scan of patient outlining the fetus. Anatomical structures clearly visible on the fetus include the calvarium, distal left upper extremity, epyphyseal growth plates of the lower extremities, and both feet

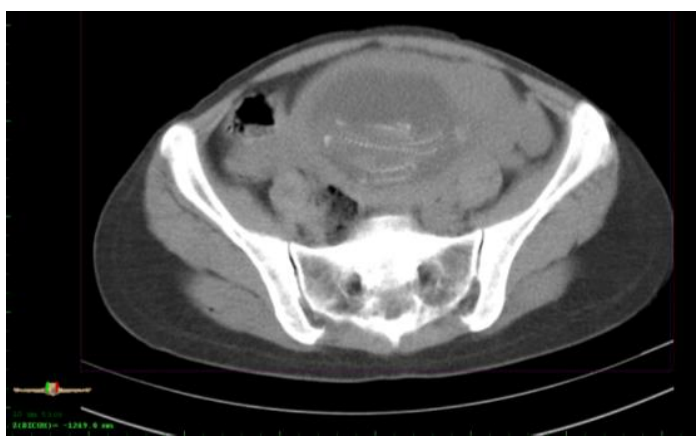

Fig. 2 CT scan clearly showing a $90^{\circ}$ rotation of the fetus around the caudo-cephalic axis. Vertebral bodies, upper extremities, and pelvic structures, are obvious 
Clearly, this fetus was unintentionally exposed to some dose of radiation and approximately two weeks later, it became non-viable. As a precaution, external beam radiotherapy was initially excluded for this patient, and instead, the non-viable fetus was removed in a modified Wertheim-Meigs procedure with curative intent. The patient then received 50 Gy of external beam photon therapy, and an additional 15 Gy of brachytherapy as a boost. She later suffered episodes of renal insufficiency, and psychoses, and expired approximately one year after initial consultation due to complications from parametrial metastases.

To address administrative as well as potential medico-legal implications, the hospital administration referred this case to the Physics Department for further dosimetric evaluation. Ethical clearance was secured from the relevant institutional committee before submitting this work for publication. The intent of the referral was to determine what role - if any - the fetal dose played in the premature termination of the pregnancy. The fetal dose was determined using Wagner's CTDI Phantom Dose Reference Model method [6]. The method is based on the use of different tabulated reference values such as an $F(0)$ value used to calculate dose in a single slice of the fetus within the primary beam, and multiple $\mathrm{F}\left(\mathrm{z}_{\mathrm{i}}\right)$ values to account for the scatter component from the remaining slices displaying the fetus. A slice thickness of $6 \mathrm{~mm}$, and a CTDI $\mathrm{vol}_{\mathrm{v}}$ of $1.13 \mathrm{mGy}$ were used in our calculations, as suggested in the Abdomen Baby manufacturer's protocol [7]. In addition, $\mathrm{kVp}, \mathrm{mAs}$, and slice thickness corrections were applied to the $\mathrm{CTDI}_{\mathrm{vol}}$, as suggested by Wagner.

\section{Results and Discussion}

With the given parameters, our estimated absorbed dose to the fetus was $19.3 \mathrm{mGy}$. We estimate that the rotation of the fetus through an angle of approximately $90^{\circ}$ along the caudo-cephalic axis during image acquisition, had no clinically relevant effect on the calculated absorbed dose (Fig. 2). The calculated dose received by the fetus is well below the average fetal dose of $30 \mathrm{mGy}$ from a CT abdominal examination [6]. Furthermore, this dose is comfortably below the consensus levels for negligible risk ( $50-150 \mathrm{mGy})$, and substantially below the "actionable" level of $150 \mathrm{mGy}$ [8]. Additionally, this fetus was approximately 12 weeks old at the time of irradiation and thus well past the most radiosensitive phase of organogenesis. Within this phase, it is the initial 10 days of pregnancy which are of most concern regarding a possible radiationinduced abortion. The "all-or-none" principle applies during this initial phase, and it establishes that the conceptus either survives relatively unscathed, or it becomes non-viable, following irradiation [9]. We note that some caution needs to be exercised when applying this principle to actual cases since it is based primarily on models from the Life Span Study (i.e. radiation-induced effects to individuals exposed in the Hiroshima and Nagasaki atomic bombings). Based on these considerations, our best estimate is that the probability of a radiation-induced abortion from this CT scan is extremely small, and can be safely ignored. Thus, the premature termination of this pregnancy can be reasonably attributed to other causes.

\section{Conclusion}

The dose absorbed to the fetus was well below both, the consensus levels for negligible risk (50-150 mGy), and the "actionable" level of 150 mGy. At the time of exposure, the fetus was developmentally beyond the critically radiosensitive phase of organogenesis. We conclude that the premature termination of this pregnancy is not likely to be of radiological etiology.

\section{References}

[1] J.P. Felmlee, J.E. Gray, M.L. Leetzow, J.C. Price, Estimated fetal radiation dose from multislice CT studies, Am. J. Roentgenol. 154(1) (1990) 185-190.

[2] L.M. Hurwitz, T. Yoshizumi, R.E. Reiman, P.C. Goodman, E.K. Paulson, et al., Radiation dose to the fetus from body MDCT during early gestation, Am. J. Roentgenol. 186(3) (2006) 871-876.

[3] Anonymous, ImPACT CT Dosimetry. Imaging performance assessment of CT scanners: a medical devices Agency evaluation group. CT scanner matching data, tables of CTDI values in air, $\mathrm{CTDI}_{\mathrm{w}}$, and phantom factor values. http://www.ImPACTscan.org (Accessed on: 08.06.2019)

[4] D.G. Jones, P.C. Shrimpton, Survey of the practice in the UK, Normalized organ doses calculated using Monte Carlo techniques, NRPB R-250, Chilton, National Radiological Protection Board, England, 1991.

[5] W.S. Snyder, H.L. Fisher, M.R. Ford, G.G. Warner, Estimates of absorbed fractions for monoenergetic photon sources uniformly distributed in various organs of a heterogeneous phantom, J. Nucl. Med. (Supp. 3) (1996) 7-52.

[6] L. Wagner, R.G. Lester, L.R. Saldana, Exposure of the pregnant patient to diagnostic radiations: A guide to medical management: $2^{\text {nd }}$ Ed., Medical Physics Publishing, Madison, WI, 1997.

[7] Anonymous, Siemens Somatom Spirit CT Application Guide, Germany, 2007.

[8] C.H. McCollough, B.A. Schueler, T.D. Atwell, N.N. Braun, D.M. Regner, et al., Radiation exposure and pregnancy: when should we be concerned?, Radio Graphics 27(4) (2007) 909-917.

[9] E. Hall, Radiobiology for the Radiologist, 5th Ed., Lippincott Williams and Wilkins, Philadelphia, PA, 2000. 